\title{
The broadening spectrum of osteoporotic vertebral fracture
}

\author{
Fergus Eoin McKiernan
}

Published online: 30 January 2009

(C) ISS 2009

\section{Introduction}

Osteoporotic bone is structurally insufficient and predisposed to low-energy fracture [1]. Fractures are deemed osteoporotic when they result from generalized skeletal demineralization and low-energy trauma rather than from focal pathologic destruction of bone or high-energy trauma. Osteoporotic fractures are accompanied by substantial morbidity and signify increased mortality [2]. Vertebral fractures, the most prevalent osteoporotic fracture, is a powerful harbinger of future vertebral and non-vertebral fracture. Since vertebrae generally fail by compression in axial loading, these fractures are often called vertebral compression fractures. Since fracture morphology is often more complex then this name suggests, the more inclusive term, vertebral fracture (VF), is preferred.

In clinical practice, osteoporotic VFs are usually identified by characteristic changes in vertebral shape on a lateral spine radiograph. For research consensus, VFs are defined by a reduction in anterior, middle, or posterior vertebral height although the minimum required reduction (e.g., $15 \%$ or $20 \%$ of vertebral height) varies among definition schemes [3]. Alterations in vertebral shape consistent with osteoporotic VF are further classified based on the presence or absence of fracture symptoms. Those associated with fracture pain are termed "clinical" while all others "morphometric" VFs (Fig. 1). This dichotomous view teaches that the majority of osteoporotic VFs are morphometric $(75 \%)$, that is, clinically silent, rather than clinical (25\%) [4]. Descriptive

F. E. McKiernan ( $\bowtie)$

Center for Bone Disease, Marshfield Clinic,

Marshfield, WI 54449, USA

e-mail: mckiernan.fergus@marshfieldclinic.org fracture morphology further characterizes VFs by shape (e.g., compression, wedge, concave, plana), severity (mild, moderate, severe), number and location within the spine. In this discussion, the term "vertebral deformity" will refer to other non-osteoporotic, non-fracture alterations of vertebral shape.

These binary fracture definition schemes have served as the basis for the epidemiology of osteoporosis, defining the economic burden of osteoporotic fractures and as therapeutic endpoints in pivotal pharmaceutical osteoporosis trials [5]. Prevalent VFs have been shown to increase the risk of future vertebral and non-vertebral osteoporotic fracture independent of bone mineral density (BMD). Further, a linear correlation exists between both the number and severity of prevalent VFs and future VF risk. These relationships are the underpinnings of vertebral fracture analysis (VFA) meant to enhance BMD-based fracture risk assessment by combining detected prevalent VFs with BMD T-scores [6]. Substantial clinical data indicates that osteoporotic VF, whether symptomatic or not, correlates with disability from back pain, reduced physical function, reduced quality of life, and increased mortality [2, 7-10]. While binary fracture definition schemes have served the osteoporosis community well, clinical experience, refined by recent conceptual advances in vertebral morphometry $[5,11$, 12], and the emerging vertebral augmentation experience [13], suggest that the spectrum of osteoporotic VFs is broader than these definition schemes suggest.

The intention of this perspective is to challenge the prevailing dichotomous view of osteoporotic VF. Some of what follows is speculation based upon published clinical and bench research of varying rigor. All of what follows is intended to stimulate a multidisciplinary discussion of a broader view of osteoporotic VFs. 


\section{Fracture symptoms? \\ $+$ \\ Vertebral height loss \\ $15 \%$ or $20 \%$ \\ $\begin{array}{cc}\text { Clinical } & \text { Morphometric } \\ \text { VF } & \text { VF }\end{array}$ \\ VF}

Fig. 1 Dichotomous view of osteoporotic vertebral fractures

\section{Can vertebral fractures be painless?}

The notion of painless fracture is counter-intuitive to most clinicians. Therefore, the assertion that the vast majority of osteoporotic VFs are painless should give us some cause for reflection. There are several straightforward reasons why VFs could be deemed "painless" (i.e., morphometric VFs) when in fact they are not. Initially painful VFs could be erroneously construed to be painless by the mere passage of time and poor recall; undoubtedly, a few to medical inattentiveness or neglect. Fracture pain may have been erroneously ascribed to more common sources of back pain such as facets or intervertebral discs. In fact, the correlation and implied causality between prevalent VF and the presence or absence of back pain is itself presumptuous, and usually incorrect. Back pain is common in osteoporotic women and neither prevalent nor incident back pain is highly predictive of prevalent or incident osteoporotic VF [7-9].

Furthermore, some changes in vertebral shape that simulate fracture (e.g., Schmorl's nodules and Cupid's bow deformity) are vertebral deformities and not osteoporotic VFs [5, 6]. Diagnostic errors may also emanate from technical shortcomings of image acquisition such as parallax or poor patient positioning. Finally, disagreement may exist even amongst expert readers. The systematic misclassification of a non-osteoporotic vertebral deformity, designated as short anterior vertebral height (SVH), may have contributed to an overestimation of the true prevalence of osteoporotic VFs $[5,11,12]$. SVH is identified by the algorithm-based quantitative (ABQ) method in which an initial qualitative judgment is made to determine the presence or absence of a vertebral deformity compatible with osteoporotic fracture. Heights of "fracture-compatible" deformities are then measured. Those deformities with $\geq 15 \%$ reduction of anterior height but which lack visible depression of the epiphyseal ring (endplate) are deemed to represent the non-osteoporotic deformity, SVH. These authors have validated the ABQ method by reanalyzing the prevalence of morphometric VFs from baseline radiographs in the MrOS trial, a large prospective study of osteoporosis in men. By excluding $\mathrm{SVH}$, the prevalence of morphometric VF at baseline was reduced by 30\% [14]. Importantly, the association between prevalent morphometric VF and low BMD was actually strengthened by excluding $\mathrm{SVH}$ in MrOS. Therefore, at least some proportion of vertebrae deemed morphometric VFs have been overdiagnosed radiographically.
In spite of this, there is still compelling evidence to indicate that osteoporotic vertebrae may fracture painlessly. In the study of 66 severely osteoporotic subjects (mean lumbar $T$-score $=-2.9$, mean number of prevalent $V F s=3$ ) Voormolen et al. [15] studied the incidence of new VF following routine vertebroplasty. Subjects were evaluated prospectively clinically for incident back pain and radiographically and by MR at baseline, 3, 6 and 12 months postoperatively. Radiography consisted of AP and lateral vertebral images. MR consisted of T1, short tau inversion recovery (STIR) and transverse T2-turbo spin echo sequences of the entire spine. The definition of incident VF required both vertebral height reduction $(>15 \%)$ and bone marrow edema (low T1, high STIR signal) by MR. One year after vertebroplasty, the per-person incident VF rate was $24 \%$. Half of all incident VFs were accompanied by VF pain and half were painless. This carefully executed, prospective study demonstrates that osteoporotic vertebrae can indeed fracture yet remain clinically silent. Similar conclusions can be extracted from a post hoc analysis of patients in the placebo arm of the Fracture Prevention Trial (FPT) a large, multicenter, prospective trial of teriparatide $\left(\right.$ Forteo $\left.^{\circledR}\right)$ versus placebo for postmenopausal osteoporosis [9]. Primary endpoints in FPT were changes in BMD and incident VF. Back pain prevalence was assessed at study entry while new or worsening back pain was captured as adverse events. Incident VF was defined as a $20 \%$ reduction in anterior, middle, or posterior vertebral height in a previously normal vertebra. In the placebo group, more than $50 \%$ of all incident VFs were unaccompanied by reports of new or worsening back pain [9]. Even moderate or severe incident VFs were accompanied by new or worsening back pain in only half of the subjects [9]. Therefore, clinically silent, radiographically confirmed VFs do occur. For the remainder of this discussion, these VFs will be referred to as true morphometric VFs.

Refining the definition of morphometric VF is worthwhile as it may result in more accurate estimates of VF prevalence and incidence. The BMD-independent relationship between prevalent VF and future fracture risk should be only stronger for true morphometric VFs. True morphometric VFs may be sufficient indication to initiate medical osteoporosis treatment [6]. In the future, true morphometric VFs might become reasonable targets when therapeutic interventions such as vertebral augmentation (e.g., vertebroplasty) can deliver skeletal anabolic agents rather than polymethylmethacrylate.

\section{Can painful vertebral fractures elude radiographic detection?}

For research purposes, VFs are defined by a minimum threshold loss of vertebral height [3]. Nevertheless, before 
meeting any arbitrary height loss threshold, a fracturing vertebra is still fracturing. Clinicians understand that VF pain may precede measurable changes in radiographic vertebral configuration by days and sometimes by weeks. This lag is the reason that imaging modalities such as nuclear scintigraphy and MR are useful in identifying symptomatic VFs before diagnostic radiographic changes can be appreciated. Radiographic lag also likely contributes to the "delay" in the diagnosis of "delayed post-traumatic osseous necrosis of the vertebral body" known eponymously as Kümmel's disease [16]. Thus, clinicians are comfortable with the concept that painful, fracturing vertebrae, particularly recently fracturing vertebrae, may be unaccompanied by diagnostic radiographic findings. Furthermore, there is evidence that vertebrae may fracture, generate fracture pain yet never result in measurable changes in radiographic configuration or height. Healed trabecular micro-fracture and fracture repair are common histologic findings in sections of fractured osteoporotic vertebrae [17]. Surprisingly, similar histologic findings are only slightly less common in contiguous, non-fractured osteoporotic vertebrae [17]. These findings support the assertion that VF, micro-fracture, and fracture repair occurs frequently even in the absence of any appreciable radiographic change in vertebral shape. Recently, Pham et al. [18] described 16 osteoporotic subjects with acute, severe back pain suggestive of VF but in whom no VF could be appreciated on lateral spine radiograph. Clinical vertebral fracturing was supported by the finding of bone marrow edema on MR and/or characteristic linear uptake on radionuclide bone scintigraphy in an anatomic distribution correlating with clinical pain. Subjects were followed-up prospectively until clinical VF pain resolved (3-18 months). At the conclusion of the study, lateral vertebral radiographic changes consistent with osteoporotic VF (Genant stage $\geq$ " 0.5 ") [3] developed in $80 \%$ of subjects. In the remaining $20 \%$, severe back pain resolved in the absence of any radiographically detectable VF. Whether radiographic VF was eventually detected or not could not be predicted by the clinical course as the two groups were otherwise identical. Therefore, clinically painful, radiographically occult osteoporotic VFs do occur. Recently, Yang et al. [19] performed early vertebroplasty in a similar group of osteoporotic patients. Bone marrow edema on MR, consistent with acute VF, supported the decision to perform early vertebroplasty in spite of normal radiographs. Vertebroplasty resulted in rapid pain relief and improved physical function similar to outcomes of vertebroplasty in classically defined osteoporotic VFs. Therefore, painful osteoporotic VFs can occur in the absence of appreciable changes in vertebral shape, can behave clinically like classically defined osteoporotic VFs yet forever elude radiographic detection. These clinically painful, radiographically unapparent fractures will be re- ferred to as "occult" VFs. In the absence of a permanent radiographic record, back pain resulting from an incident occult VF will be erroneously attributed to other anatomic causes.

At the baseline enrollment examination for the Study of Osteoporotic Fractures (SOF), 68\% of postmenopausal women aged $65-70$ years $(n=2992)$ reported experiencing back pain within the previous year [7]. Of those reporting pain, $64 \%$ rated that pain as moderate to severe and $22 \%$ reported that their back pain was present most or all of the time. Back pain prevalence, intensity, and persistence were similar whether a morphometric VF could be appreciated radiographically or not. Similar results were found in women at the baseline examination for the FPT who harbored Grades 1 and 2 morphometric VFs [8]. Therefore, while prevalent and incident back pain is common in postmenopausal osteoporotic women, it neither necessarily indicates the presence or absence of a morphometric VF nor confidently allows assignment of causality [7-10]. In light of these findings and extrapolating from the observations made by Yang et al. [19], it seems plausible that a substantial number of women in both the SOF and FPT actually experienced a painful, radiographically occult VF but had their pain misconstrued to be non-fracture back pain. Some proportion of occult VFs evolve to become classic clinical VFs as vertebral configuration changes and becomes radiographically apparent. Thus, the true prevalence of occult VFs and the factors that determine their transition to clinical VFs are unknown. Whether occult VFs bear prognostic information similar to clinical or morphometric VFs is also unknown, although this would seem reasonably likely.

Clinical management of occult VFs has rarely been discussed. It would seem reasonable to consider a lowenergy occult VF, when recognized, as sufficient indication to initiate medical osteoporosis therapy independent of BMD results. When occult VFs occur within biomechanically vulnerable or stress concentrating segments of the spine (such as the thoraco-lumbar junction) a strong biomechanical argument could be made for early and aggressive bracing. Theoretically, by mitigating anterior compressive loads, the number of occult VFs that become clinically apparent might be reduced. In the future, occult VFs at risk for becoming clinically significant might benefit from injection with mechanically competent, resorbable biomaterials.

\section{Can vertebral fractures occur in the absence of both symptoms and $\mathrm{X}$-ray changes?}

If vertebrae can fracture and change shape in the absence of symptoms and if painful fracturing can occur in the absence of radiographic detection, it seems at least plausible that 
vertebrae could fracture in the absence of both fracture symptoms and X-ray changes. In the prospective study of Voormolen et al. [15] previously discussed (personal communication) and in clinical practice, transient vertebral marrow edema events that fail to meet a radiographic threshold for fracture and remain clinically silent are not uncommon. That altered MR vertebral marrow signal is not simply "bone edema" is supported by the histopathologic findings of trabecular fracture and repair in "unfractured" osteoporotic vertebrae [17]. These clinically and radiographically unapparent osteoporotic VFs will be termed silent osteoporotic VFs. Undoubtedly, many initially silent VFs will become symptomatic in the absence of X-ray changes (occult), or with X-ray changes (clinical) or may become radiographically apparent in the absence of symptoms (true morphometric). The prevalence, prognostic value, clinical significance, and "management" of silent VFs is entirely unknown and, heretofore, never been discussed. If those silent VFs destined to become clinical VFs could be identified early, then the injection of locally retained anabolic material might be an attractive intervention in the future. Until the significance of silent VFs is better understood, they will remain incidental findings sometimes reported by radiologists but more often not. Without known significance, it would be difficult to justify looking for silent VFs.

\section{What about Kümmel's disease?}

Kümmel's disease is the eponym given to "delayed posttraumatic osseous necrosis of the vertebral body" $[13,16]$. The radiographic hallmark of Kümmel's disease is the intravertebral vacuum cleft and the pathophysiology is commonly accepted to be aseptic necrosis of vertebral bone. Contemporary radiologic and clinical reviews continue to promote the notion that Kümmel's disease is a rare event and not within the usual spectrum of osteoporotic VF events [20]. The vertebral augmentation experience suggests otherwise. When sought, intravertebral vacuum clefts are actually common in symptomatic, fracturing, osteoporotic vertebrae. One third of vertebrae in patients presenting for vertebroplasty contained intravertebral vacuum clefts [13]. Intravertebral clefts typically lie beneath the superior endplate in the anterior portion of the vertebral body and are most readily demonstrated at the thoraco-lumbar junction [16]. Their presence implies complete corticocancellous disruption of bone and clefts fill as confluent reservoirs for polymethylmethacrylate (PMMA) at vertebroplasty. Intravertebral clefts permit dynamic mobility, the VF property that can be harnessed to effect vertebral height restoration at the time of vertebroplasty [13]. In retrospect, the body of Kümmel's literature affirms that most reports occur in older adults with osteoporosis, in women more often than men and typically at the thoraco-lumbar junction. There is even an early demonstration of a cleft being elicited by dynamic radiography. By definition, these painful VFs eluded early radiographic detection and would thus have initially been considered an "occult" VF that later became radiographically apparent and finally recognized as a severe clefted, likely dynamically mobile VF [16]. The delay in the "post-traumatic, delayed osseous necrosis" thus had more to do with the rigor of diagnostic ascertainment than with any specific pathophysiology.

The tendency for dynamic mobility and clefts (Kümmel's disease) to affect the thoraco-lumbar junction reflects spinal biomechanics and stress concentration at this location. That intravertebral clefts typically lie beneath the superior endplate is consistent with detailed anatomic studies conducted by Vernon-Roberts in the last century [21]. Healing micro-fractures within osteoporotic lumbar vertebrae overwhelmingly affected vertical trabecular struts and were concentrated beneath the vertebral endplates. More recently, texture and complex finite element analyses have shown that the initial and most highly strained region of the vertebra lies beneath the superior endplate in axial loading $[22,23]$. The biologic explanation for this observation may lie in the demonstration of zone-dependent changes in vertebral trabecular structure with normal aging. In a crosssectional analysis of human cadaveric vertebrae from the third through ninth decade Thomsen et al. [24] demonstrated that the sub-endplate zone showed greater reductions in trabecular volume and connectivity density and greater increases in star-volume compared with the central vertebral zone. Thus, more rapid turnover within bone immediately beneath the vertebral endplate results in bone that is more osteoporotic and thus likely to fracture. Structural failure at the spinal segment subjected to the greatest stress concentration likely begets more structural failure and necrosis. In its most severe form, Kümmel's disease represents catastrophic failure of the entire vertebral body. The true prevalence of Kümmel's disease is unknown but the frequent elicitation of dynamic mobility in the vertebroplasty population suggests that it is far more common that has previously been appreciated. The prognostic significance of Kümmel's disease is also unknown but since fracture severity correlates with future VF risk Kümmel's disease likely represents a poor prognostic event.

Clinical management of Kümmel's disease has only recently been discussed and usually in the context of vertebral augmentation. Painful, clefted, dynamically mobile VFs respond well to vertebral augmentation but may be associated a higher risk of post-procedural adjacent VF. Large clefts and grossly unstable VFs pose a substantial biomechanical challenge. Currently, only PMMA and a few calcium-phosphate cements are available for intravertebral injection at vertebroplasty. Compacted morcelized bone 
autografted in to the cleft might provide immediate structural stability while offering the possibility of future remodeling and full incorporation of the autograft into the vertebra.

\section{A broader view of osteoporotic VFs}

Osteoporotic VFs are more heterogeneous than our current binary classification scheme suggests. The dichotomous "clinical" versus "morphometric" view does not capture the full spectrum of VFs encountered in clinical practice, limits our understanding of clinical osteoporotic fracture and may be constraining us from recognizing new horizons in the management of patients with osteoporosis and VF. Vertebral shape is plastic to some degree and changes with mechanical loading, posture and over time. A wider view of the spectrum of osteoporotic VFs is presented in Fig. 2. In this view, VF events are evolving rather than completed anatomic events. On one end of the spectrum are clinically and radiographically silent, microscopic, trabecular fracture/repair events. On the other end of the spectrum are tissue necrosis, macroscopic clefts, vertebral instability, and clinical incapacitation. In this view, Kümmel's disease represents the most catastrophic form of vertebral failure rather than a distinct clinical entity. The eponym "Kümmel's disease" should be retired from clinical use. There are immediate practical implications that flow from recognizing a broader spectrum of osteoporotic VFs. At the very least, mindful that painful VFs are not always accompanied by radiographic changes, fewer frail elderly patients should be dismissed from urgent and emergency care settings with misdiagnoses of non-specific back pain, muscle strain, or pleurisy. Emphasizing the evolutionary nature of osteoporotic VFs might prompt earlier reevaluation of persistent back pain when the initial radiographic evaluation is negative. Furthermore, radiographic imaging for the elderly presenting with acute back pain might be more fruitful and certainly less painful if a single standing (axial loaded) lateral radiograph, centered on the clinical region of interest, were routine. Current standard radiology protocols perform AP and lateral spine radiographs in the supine and lateral decubitus positions, respectively. This positioning is difficult and painful for frail older adults who have fractured. Both of these images are axially un-loaded and risk missing subtle dynamic endplate depressions that might be obvious on axially loaded (standing lateral) views. If silent or occult VFs bear prognostic significance then they might provide an independent indication for therapeutic intervention. Perhaps radiology services should develop quick, cost-effective protocols for recognition of occult VF in urgent care areas such as a limited, sagittal STIR sequence of the relevant spinal region of interest.

In the field of vertebral augmentation, the recognition of diverse fracture types will help determine the most appropriate composition and biomechanical properties of injectable materials and biomaterials. Infiltration pressure, trabecular permeability and extravasation risks within a silent VF are not the same as those of a clinical VF. The structural requirements of materials placed into a severe, clefted, dynamically mobile VF will be different than those of a painful occult VF. Currently available materials (PMMA) are inexpensive and inert but forever preclude the possibility of intravertebral trabecular remodeling and mature fracture repair. While this may be acceptable in older patients, it is less desirable in younger adult patients and unacceptable in children. Newly developed calcium-phosphate cements may provide immediate structural stability while allowing resorption and eventual trabecular reconstitution but are costly. In the future, injected and locally retained anabolic agents may be viable interventions for silent VFs or even preemptive interventions in high-risk non-fractured vertebrae such as those sandwiched between augmented vertebrae. A similar argument could be made for preemptive bracing.

Much of what is known about the epidemiology of osteoporosis and the efficacy of our current osteoporosis treatments is based on the visual identification of VFs. The dichotomous distinction between clinical VF and morphometric VF has been extremely helpful but may no longer be sufficient. Little is known and much must be learned about the incidence, prevalence, proper diagnosis, prognostic

Fig. 2 A broader spectrum of clinical osteoporotic vertebral

Vertebral fracture event fractures

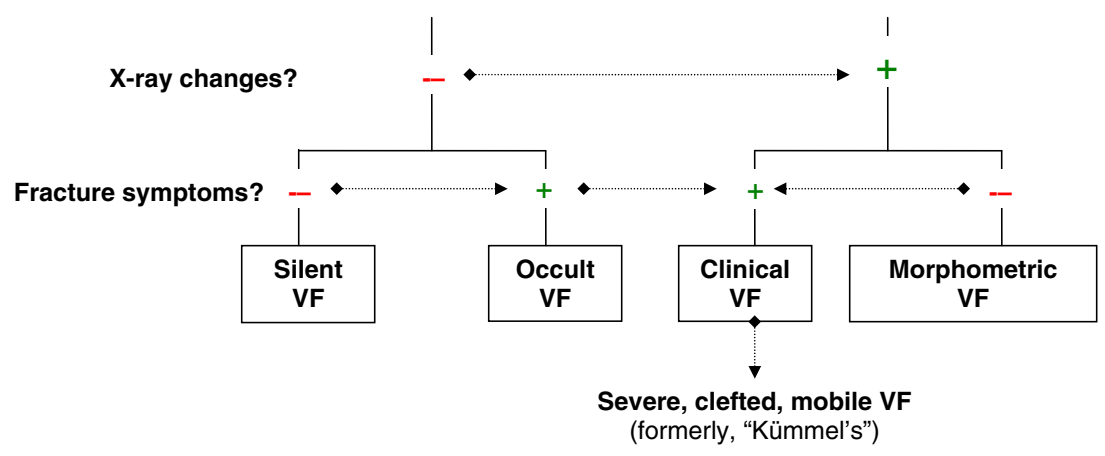


significance, and implications for treatment of the different fracture types proposed herein. Broadening the clinical spectrum of osteoporotic VF and understanding its natural history better should empower clinicians to better manage this condition in its varied presentations.

\section{References}

1. Seeman E, Delmas PD. Bone quality - the material and structural basis of bone strength and fragility. NEJM 2006; 354: 2250-2261.

2. Lips P, van Schoor NM. Quality of life in patients with osteoporosis. Osteoporos Int 2005; 16: 447-455.

3. Genant HK, Wu CY, van Kuijk C, Nevitt MC. Vertebral fracture assessment using a semiquantitative technique. J Bone Miner Res 1993; 8: 1137-1148.

4. Fink HA, Milavetz DL, Palermo L, Nevitt MC, Cauley JA, Genant HK, Black DM, Ensrud KE, Fracture Intervention Trial Research Group. What proportion of incident radiographic deformities is clinically diagnosed and vice versa? J Bone Miner Res 2005; 20: 1216-1220.

5. Ferrar L, Jiang G, Adams J, Eastell R. Identification of vertebral fractures: an update. Osteoporos Int 2005; 16: 717-728.

6. Schousboe JT, Vokes T, Broy SB, Ferrar L, McKiernan F, Roux C, Binkley N. Vertebral fracture assessment: the 2007 ISCD official positions. J Clin Densitom. 2008; 11: 92-108.

7. Ettinger B, Black DM, Nevitt MC, Rundle AC, Cauley JA, Cummings SR, Genant HK. Contribution of vertebral deformities to chronic back pain and disability. The Study of Osteoporotic Fractures Research Group. J Bone Miner Res 1992; 7: 449-456.

8. Crans GG, Silverman SL, Genant HK, Glass EV, Krege JH. Association of severe vertebral fractures with reduced quality of life: reduction in the incidence of severe vertebral fractures by teriparatide. Arthr Rheum 2004; 50: 4028-4034.

9. Genant HK, Halse J, Briney WG, Xie L, Glass EV, Krege JH. The effects of teriparatide on the incidence of back pain in postmenopausal women with osteoporosis. Curr Med Res Opin 2005; 21: 1027-1034.

10. Roux C, Priol G, Fechtenbaum J, Cortet B, Liu-Leáge S, Audan $\mathrm{M}$. A clinical tool to determine the necessity of spine radiography in postmenopausal women with osteoporosis presenting with back pain. Ann Rheum Dis 2007; 66: 81-85.
11. Jiang G, Eastell R, Barrington NA, Ferrar L. Comparison of methods for the visual identification of prevalent vertebral fractures in osteoporosis. Osteoporos Int 2004; 15: 887-896.

12. Ferrar L, Jiang G, Clowes JA, Peel NF, Eastell R. Comparison of densitometric and radiographic vertebral fracture assessment using the algorithm-based qualitative (ABQ) method in postmenopausal women at low and high risk of fracture. J Bone Miner Res 2008; 23: $103-111$.

13. McKiernan FE, Faciszewski T, Jensen R. The dynamic mobility of vertebral compression fractures. J Bone Mineral Res 2003; 18: 24-30.

14. Ferrar L, Jiang G, Cawthon PM, San Valentin R, Fullman R, Lambert L, Cummings SR, Black DM, Orwoll E, Barrett-Connor E, Ensrud K, Fink HA, Eastell R. Identification of vertebral fracture and non-osteoporotic short vertebral height in men: the MrOS study. J Bone Miner Res 2007; 22: 1434-1441.

15. Voormolen MH, Lohle PN, Juttmann JR, van der Graaf Y, Fransen H, Lampmann LE. The risk of new osteoporotic vertebral compression fractures in the year after percutaneous vertebroplasty. J Vasc Interv Radiol 2006; 17: 71-76.

16. McKiernan FE, Faciszewski T. Intravertebral clefts in osteoporotic vertebral compression fractures. Arth Rheum 2003; 48: 1414-1419.

17. Antonacci MD, Mody DR, Rutz K, Weilbaecher D, Heggeness MH. A histologic study of fractured human vertebral bodies. J Spinal Disord Tech 2002; 15: 118-26.

18. Pham T, Azulay-Parrado J, Champsaur P, Chagnaud C, Legré V, Lafforogue P. "Occult" osteoporotic vertebral fractures: vertebral body fractures without radiologic collapse. Spine 2005; 30: 2430 2435.

19. Yang X, Mi S, Mahadevia AA, Lin X, Shi W, Liu A, Li L, Wu Z, Murphy K. Pain reduction in osteoporotic patients with vertebral pain without measurable compression. Neuroradiol 2008; 50: 153-159.

20. Theodorou D. The intravertebral vacuum cleft sign. Radiology 2001; 221: 787-788.

21. Vernon-Roberts B, Pirie CJ. Healing trabecular microfractures in the bodies of lumbar vertebrae. Ann Rheum Dis 1973; 32: 406-412.

22. Bay BK, Yey SA, McLain RF, Toh E. Measurement of strain distributions within vertebral body sections by texture correlation. Spine 1999; 24: 10-17.

23. Thomsen JS, Ebbesen EN, Moskilde LI. Zone dependent changes in human vertebral trabecular bone: clinical implications. Bone 2002; 30: 664-669.

24. Eswaran SK, Gupta A, Keaveny TM. Locations of bone tissue at high risk of initial failure during compressive loading of the human vertebral body. Bone 2007; 41: 733-739. 